\title{
REFLEXIÓN DE LA RELACIÓN QUE EXISTE ENTRE LA PRONUNCIACIÓN, LA COMPRENSIÓN AUDITIVA Y LA EXPRESIÓN ORAL EN LA LENGUA INGLESA
}

\author{
REFLECTION OF THE RELATIONSHIP BETWEEN PRONUNCIATION, \\ LISTENING AND SPEAKING IN ENGLISH
}

\author{
Iliana Real Poveda, MAE \\ Docente Universidad Estatal de Milagro, UNEMI \\ ilianarealpoveda@gmail.com \\ Irlanda Maridueña Macancela, MAE \\ Docente Universidad Estatal de Milagro, UNEMI
}

\section{RESUMEN}

Una de las principales debilidades al comunicarse de forma oral en inglés, es el nivel fonético bajo o la dificultad de pronunciación, causando en el discente: desmotivación, frustración e incluso una visión equivocada de su valor como ente que necesita interactuar y compartir en una sociedad tan competitiva, donde quienes han desarrollado sus competencias y habilidades son los candidatos idóneos para aprovechar las oportunidades académicas, laborales y sociales que se presenten. Esta investigación se desarrolló bajo el paradigma cualitativo, a través de un estudio de caso único, de tipo factual, con 31 estudiantes del segundo semestre 2014 de la Facultad de Ciencias de la Ingeniería, de la Universidad Estatal de Milagro (UNEMI). En este caso de estudio se dio énfasis al acompañamiento total del docente como punto de partida (starting point) seguido de la evaluación-detección de debilidades, trabajo autónomo específico, de acuerdo al nivel de estudio de los estudiantes y evaluación oral final que medirá lo adquirido, en gran parte, por el trabajo autónomo de los alumnos para comprobar que es posible reforzar el aprendizaje del inglés como lengua extranjera en un espacio adicional al salón de clases y sin la total presencia del docente.

Palabras clave: Pronunciación - comprensión auditiva - expresión oral - lengua inglesa

\begin{abstract}
One of the major weaknesses in communicating orally in English which is studied as a foreign language is the low phonetic level or difficulty to pronounce correctly in that language, it causes in the learner lack of motivation, frustration and even a mistaken view of its value as a human being that needs to interact and share in a highly competitive society, where those who have developed their skills and abilities are ideal candidates to take advantage of the academic, employment and social opportunities that arise. This research was conducted under the qualitative paradigm, through a single case study of factual type, with 31 students from the State University of Milagro (UNEMI) of the Faculty of Engineering of the second semester 2014. The authors claim that teaching strategies should be established in the classroom and beyond to teach and learn another language. This case study emphasizes the full support of teachers as a starting point followed by evaluation-detecting weaknesses, specific autonomous work according to the students' level of study and final oral evaluation to measure what was acquired mainly because of students' independent work to ensure that it is possible to reinforce the learning of English as a foreign language in a different place from the classroom without the presence of the teacher.
\end{abstract}

Key words: Pronunciation - Listening comprehension - oral expression - English language

Recibido: enero de 2016 Aprobado: mayo de 2016

\section{Introducción}

El aprendizaje del inglés como lengua extranjera es un proceso sencillo cuando empieza a edad temprana y en un medio expuesto siempre o con frecuencia al mismo, sin embargo se torna más complicado a edad adulta por ejemplo a nivel universitario por distintas razones, siendo una de las principales debilidades entre los alumnos, la producción de fonemas o sonidos, lo que comúnmente se conoce 
como Pronunciación. Jones, D (1967) citado por Cieri L y Barbeito M (1) enuncia cinco aspectos que obstaculizan al alumno cuando intenta aprender a hablar un nuevo idioma: Reconocer los sonidos de la lengua extranjera, producirlos, usarlos adecuada y correctamente en cuanto a su distribución en el habla enlazada, usarlos adecuadamente en cuanto a sus prosodias (especialmente acento, tono de voz, ritmo y entonación) y concatenar los sonidos en una secuencia. Para Latham-Koening C, Oxenden C y Seligson P (2013) (2) los estudiantes de nivel principiante necesitan una base sólida en los sonidos del inglés, desarrollo de pronunciación específica y ver donde hay reglas y patrones.

A opinión de las autoras, la cuasi perfección de la pronunciación de los sonidos de la lengua inglesa puede conseguirse con tres claves: la observación, repetición, y motivación de querer hablar bien el idioma; la memoria podría ser de gran ayuda para almacenar momentos importantes asociados con la fonética inglesa; por ejemplo, una palabra que llame la atención del alumno, cualquiera que sea el motivo, puede estar incluida en el nuevo fonema que el docente está introduciendo a su grupo de estudiantes. (3) La observación, primera clave mencionada por las autoras, puede estar luego seguida de una gran estrategia que al mismo tiempo es sencilla de aplicar: Escribir debajo de las letras los fonemas que les corresponde si al alumno se le complica recordar cómo se pronuncia aquella letra o sílaba en inglés. Al hacerlo por repetidas ocasiones, el resultado será la pronunciación correcta de una nueva palabra en inglés (4). Lo más importante será que al producir bien los fonemas la comunicación es más clara y la satisfacción de quien habla es un gran motivo para continuar con el aprendizaje o perfeccionamiento de los fonemas ingleses.(5)

Definitivamente, la exposición frecuente a la lengua extranjera es un factor vital para que la parte cognitiva tenga presente la pronunciación de palabras, sea cual fuere el idioma que se está aprendiendo. Asimismo, es importante desterrar este mito: Las habilidades de aprendizaje no se deterioran necesariamente con la edad sino que declinan por efecto de un uso irregular e infrecuente de las mismas o por efecto de una imagen negativa sobre los propios aprendizajes intelectuales (Leclerc P, 1985 citado por Cieri L y Barbeito M).(1)

Además, si se quiere que los estudiantes hablen en inglés con confianza los docentes necesitan enfocarse en Pronunciación como una de las herramientas necesarias (Oxenden C, Latham-Koening C, y Seligson P, 2008) (6). Sin embargo, en la mayoría de los casos, a pesar de tener una ex- celente pronunciación o producción de los fonemas, el acento, sobre todo en los jóvenes y adultos, evidencia quién es un nativo angloparlante y quién no. (7) Vale la pena resaltar que el acento tónico y la entonación son importantísimos porque brindan naturalidad al idioma y por consiguiente lo embellecen, mas esto no impide totalmente que en las primeras etapas del aprendizaje de la lengua inglesa donde muchas veces hay que "pensar para hablar en inglés" y los ya nombrados acento tónico y entonación no se los incorpore al hablar como lo merecen se consiga el objetivo de cualquier interacción entre dos o más personas que se están comunicando en inglés de forma oral: la comunicación o comprensión de los mensajes.(8)

Otro factor que incide en la motivación de pronunciar bien una lengua extranjera, es cuán identificado está el alumno con el país o países que la tienen como lengua materna. Indudablemente, debe existir agrado por la cultura de dichos países, tal vez su historia, política actual, situación geográfica, pues todo esto ayudará directa o inconscientemente a que los interesados en aprender el inglés como lengua extranjera, lleven su respectivo proceso con entusiasmo y compromiso (9). Celce Murcia (1991) citado por Cieri L y Barbeito M (1), considera que las variables que impiden o facilitan la adquisición de una pronunciación similar a la de un nativo son: L1, edad, exposición a la lengua extranjera, identidad, habilidad fonética innata, actitud, motivación e interés por la pronunciación. (10)

En el aula de clases, por repetidas ocasiones se ha mencionado, independientemente de la asignatura que se esté impartiendo o recibiendo, se exige una atmósfera o ambiente de empatía entre alumno-profesor, alumno-alumno, para que los procesos de enseñanza-aprendizaje sean exitosos, sumado a esto si el facilitador está preparado y transmite sus conocimientos con metodología y didáctica adecuadas, EN opinión de las autoras, el alumno que realmente desee aprender, en este caso la lengua inglesa, encontrará todos los motivos y razones para hacerlo aunque haya aspectos que no sean de su agrado dentro del entorno donde se desarrolle el aprendizaje, sean estos por ejemplo, poca socialización entre pares, incomodidad en el espacio asignado como aula, relación lejana con el docente, entre otros; es decir, quien desea aprender canaliza toda su energía hacia esa dirección y no se desanima fácilmente o por mucho tiempo para continuar en alcanzar su objetivo (11).

\section{Objetivos}

El objetivo de esta investigación es demostrar que 
el trabajo en conjunto del docente y alumnos dentro del salón de clases, así como autónomo específicamente por parte del aprendiente, pueden alcanzar que en un periodo definido de tiempo, los estudiantes adquieran la primera parte del nivel elemental de Inglés general (A1) en cuanto a la competencia fónica de acuerdo al Common European Framework of Reference (CEFR)(12). Para el desarrollo de este objetivo general se definieron como específicos de esta investigación:

a) Analizar dos preguntas del examen del primer parcial de Inglés que evaluaban pronunciación, tomadas a los alumnos de primer semestre de Ingeniería en Sistemas, muestra de esta investigación.

b) Evaluar el trabajo autónomo, realizado por el alumno, del nivel de inglés que se estudiaba con el propósito de desarrollar la destreza auditiva.

c) Comprobar la conexión directa que existe entre la pronunciación, comprensión auditiva y expresión oral desde el inicio de la adquisición del idioma Inglés como lengua extranjera al momento de producir oralmente.

d) Determinar la competencia fónica real adquirida por los estudiantes al culminar los cuatro procedimientos que son parte de la estrategia presentada por las autoras en este trabajo de investigación.

\section{Materiales y Métodos}

Se ha empleado el estudio de caso único definido por Denny (1978, p.370)(13) como "un examen completo e intenso de una faceta, una cuestión o quizás los acontecimientos que tienen lugar en un marco geográfico a lo largo del tiempo"; para esta investigación convergerán, de igual manera, los planteamientos de Patton (1980) quien lo considera como una forma particular de recoger, organizar y analizar los datos.(14)
Se considera particularista, pues el estudio se centra en un grupo de estudiantes universitarios cursando el nivel elemental de Inglés A1, teniendo un nivel descriptivo, heurístico e inductivo. También es de tipo factual según Guba y Lincoln (1981, p.374) (15) e intrínseco (Stake, 1994) (16) pues pretende alcanzar una mejor comprensión de las estrategias de aprendizaje empleadas y llevadas a cabo por los estudiantes. Se planteó desde la modalidad observacional ya que es la principal técnica de recogida de datos.

La estrategia didáctica propuesta en los procesos de enseñanza y aprendizaje del idioma Inglés general, en este caso en el nivel elemental A1, para demostrar la conexión directa de la pronunciación, la escucha y el habla es detallada a continuación a través de cuatro procedimientos o etapas siendo estos: a) Presentación y práctica de los fonemas durante la primera fase del nivel elemental de Inglés general A1 en el aula de clases,

b) Evaluación a través de reactivos de la primera fase del nivel elemental de Inglés general A1 para recolección y análisis de la información (detección de debilidades), c) Implementación del trabajo autónomo con tareas específicas de listening (destreza auditiva) en la segunda fase del nivel elemental de Inglés general A1 para desarrollar la escucha, y d) Evaluación oral final para análisis de la total competencia fonética adquirida durante el nivel elemental de Inglés general A1; al llegar al cuarto procedimiento enunciado, donde el alumno produce a través del habla, el docente puede evidenciar en su totalidad, porque escucha al estudiante, cuán avanzada está su pronunciación de acuerdo al nivel de inglés que está cursando, el profesor puede determinar cuánto listening ha practicado el estudiante como trabajo autónomo previo a su examen oral.

Los cuatro procedimientos han sido incluidos en una línea imaginaria del tiempo y graficados de la siguiente manera:

\section{Figura 1 Procedimiento de la actividad considerando la línea imaginaria del tiempo}

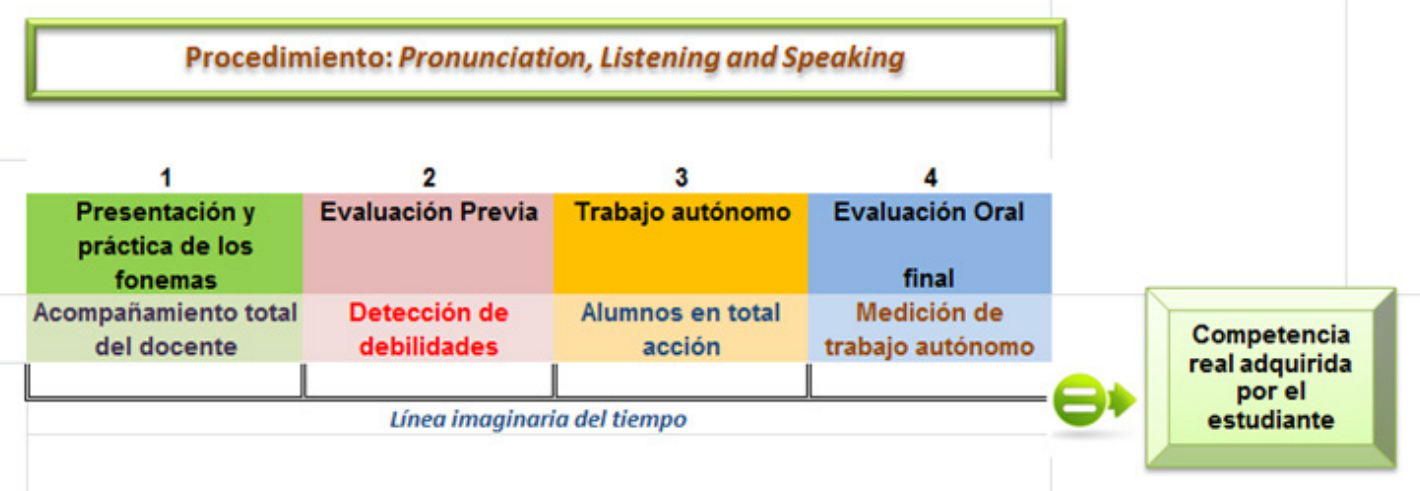


En la Universidad Estatal de Milagro (UNEMI) se toman exámenes de inglés dos veces en el transcurso del semestre, que dura aproximadamente cinco meses. A mitad del semestre, el primero (Midtermexam) que abarca todo el contenido estudiado en ese espacio, por lo general la mitad del sílabo programado y al final del mismo un segundo (Final exam) que evalúa o mide el resto del programa de estudio de la materia de inglés. (17)

El análisis que se va a realizar a continuación está basado en dos preguntas relacionadas con la fonética inglesa, es decir se examinó con esta pronunciación, de un midtermexam tomado a treinta y dos alumnos de Primero de Ingeniería en Sistemas de la sección matutina en el mes de diciembre 2014. Se escogió este grupo de estudiantes, porque a través de las observaciones hechas en sus clases se consideró que a estos alumnos les agradaba la pronunciación, veían con interés el hecho que la docente escribía debajo de las sílabas o letras, que ella consideraba pertinente, los fonemas ingleses con el propósito que ellos recuerden, memoricen con más facilidad cómo se pronuncian tales palabras en inglés. (18)

Se consideró a los integrantes de Primero de Ingeniería en Sistemas porque son futuros profesionales directamente ligados a la tecnología por su vocación, donde el idioma por excelencia es el inglés, por tanto, deben en su momento, dominarlo. Sin embargo, a pesar de la necesidad imperiosa que tiene un futuro profesional ligado a las tecnologías de la información de ser o convertirse en bilingüe, para los alumnos de Ingeniería en Sistemas, cumplir con mayor compromiso con las otras asignaturas de su respectiva carrera es más imperioso que hacerlo con la asignatura de inglés (19). Las autoras lo han observado a lo largo de su experiencia como docentes en los diversos grupos de alumnos de Ingeniería en Sistemas de la Universidad Estatal de Milagro, esta carrera pertenece a la Facultad de Ciencias de la Ingeniería en la cual cumplir con los cuatro módulos reglamentarios de Inglés (quince créditos) previos a la obtención del título de tercer nivel, es vital, por tanto el desarrollo de la competencia fonética desde Inglés General elemental A1 es una base necesaria y ejemplar para la continuación de los siguientes módulos de Inglés,(20) por tanto está totalmente integrada al desempeño académico profesional de los estudiantes de esta carrera.

Retomando el tema del examen del primer parcial (midterm) se evaluó a través de dos reactivos con diferentes preguntas debido a la cantidad de alumnos y por consiguiente para aumentar el grado de dificultad que evite el plagio entre ellos. Dieciséis alumnos tuvieron un tema de pronuncia- ción y los otros dieciséis, uno diferente. El primer tema consistió en enlazar las palabras que compartan el mismo fonema vocal, fueron cinco ítems de los cuales ocho alumnos tuvieron tres respuestas correctas, siete estudiantes acertaron en cinco respuestas y un alumno tuvo solo una respuesta correcta. Lo expresado se resume en la siguiente tabla:

Tabla 1. Descripción de la cantidad de estudiantes y su número de respuestas correctas en relación a enlazar palabras que contengan el mismo fonema vocal.

\begin{tabular}{|c|c|}
\hline Número de alumnos & Número de respuestas correctas \\
\hline 8 & 3 \\
\hline 7 & 5 \\
\hline 1 & 1 \\
\hline 16 & 9 \\
\hline
\end{tabular}

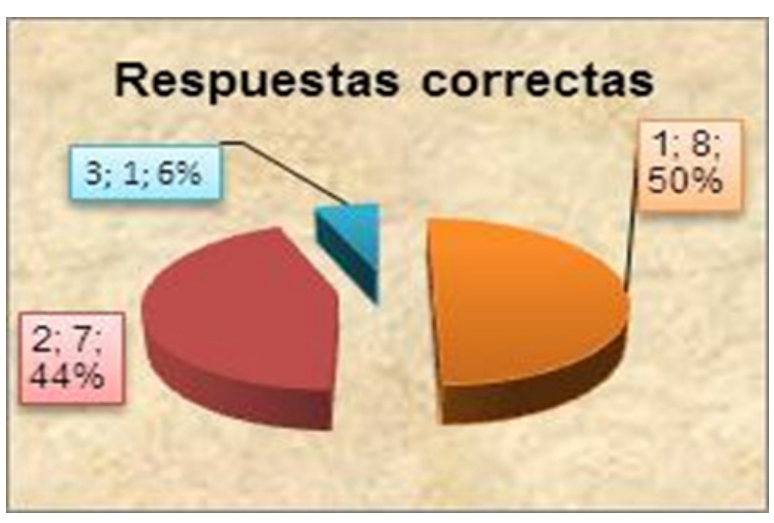

Gráfico 1.- Respuestas correctas en relación a enlazar palabras.

Se puede concluir que este grupo de estudiantes está asimilando el tema de la fonética inglesa correspondiente al nivel elemental, esta base está sentada porque solo un estudiante fracasa en esta parte de la evaluación que está representado por el 6,25\%, siete alumnos llegaron a la excelencia al responder correctamente los cinco ítems representados por el $43,75 \%$ y ocho educandos acertaron con tres ítems y esto se lo puede representar con el $50 \%$. En conclusión, quince alumnos buenos de dieciséis, es una cantidad respetable. Probablemente si los conocimientos adquiridos en cuanto a pronunciación de los otros dieciséis educandos hubieran sido evaluados con esta pregunta, los resultados serían los mismos.

El segundo tema se basó en ubicar los verbos conjugados en tercera persona del singular en presente simple, de acuerdo a cómo se pronuncian. Es menester, recalcar, que estas formas verbales tienen tres variantes fonéticas en inglés al final de la palabra: /s/, /z/ e /iz/, fueron diez verbos, 
de los cuales cinco alumnos tuvieron dos aciertos, tres estudiantes tuvieron seis respuestas correctas, dos educandos acertaron en tres ítems, dos alumnos en cuatro, un estudiante respondió con una respuesta correcta, 1 educando tuvo ocho aciertos y solo dos personas fracasaron en esta pregunta. Lo expresado se resume en la siguiente tabla:

Tabla 2. Descripción de la cantidad de estudiantes y su número de respuestas correctas en torno a la ubicación de verbos conjugados en tercera persona acorde con la pronunciación.

\begin{tabular}{|c|c|}
\hline Número de alumnos & Número de respuestas correctas \\
\hline 5 & 2 \\
\hline 3 & 6 \\
\hline 2 & 3 \\
\hline 2 & 4 \\
\hline 1 & 1 \\
\hline 1 & 8 \\
\hline 2 & 0 \\
\hline 16 & 24 \\
\hline
\end{tabular}

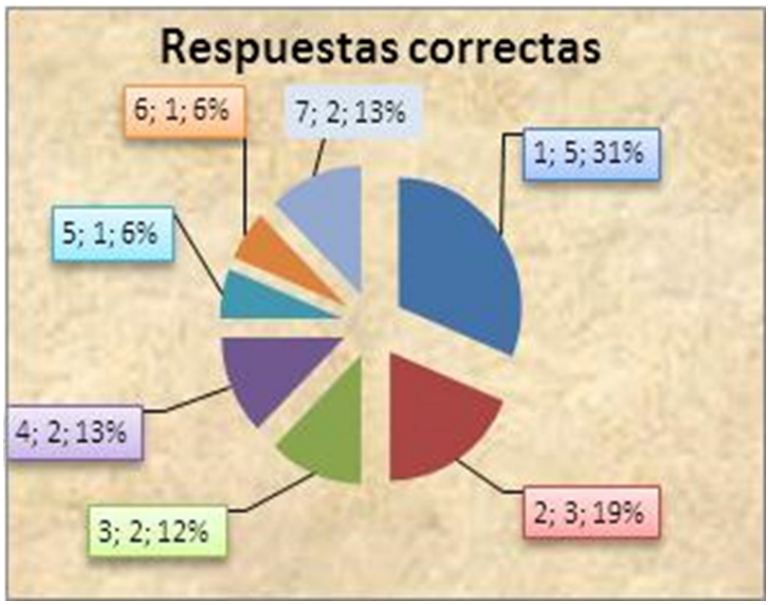

Gráfico 2.- Respuestas correctas en relación a verbos en terceras personas.

Se puede concluir que para este grupo de estudiantes no está asimilado este tema de la fonética inglesa correspondiente al nivel elemental, porque solo un alumno tiene ocho de diez aciertos representado por el $6,25 \%$, mientras que los otros quince están, a criterio de las autoras, entre lo regular e insuficiente. En conclusión, quince alumnos con estos vacíos en cuanto a sus conocimientos de esta parte de la fonética inglesa es motivo para que la docente trate de reforzar estos temas cuando la oportunidad se lo permita, para colaborar con el aprendizaje de sus alumnos y al mismo tiempo, retroalimentar en ellos esta arista de la lengua inglesa, quienes al mismo tiempo de- ben motivarse por reforzar este importante tema de pronunciación con el propósito de comunicarse en inglés con mayor claridad, cuando sea necesario y dentro del plano académico, obtener el mejor de los puntajes.

Las primeras semanas del segundo parcial, este grupo de estudiantes de Primero de Ingeniería en Sistemas trabajó en el salón de clases junto a la docente, como se lo hizo en el primer parcial, los ejercicios de listening (destreza auditiva) que correspondían, según el texto utilizado. Sin embargo, llegó la hora de cambiar la estrategia o metodología, los alumnos tuvieron como tarea y responsabilidad realizar de forma autónoma en el laboratorio de inglés de la UNEMI los ejercicios de listening; así como podían sentir estas tareas como una obligación de parte de la asignatura de inglés o extrema exigencia de la facilitadora, también fue la oportunidad de concentrarse en esta actividad para desarrollar la destreza auditiva y empezar a descubrir que pueden estudiar solos con ejercicios y temas acordes a su nivel; este tipo de actividades, a criterio de las autoras, es un reto tanto para el estudiante, quien necesita afirmarse, sentir y comprobar que puede hacerlo, como para el profesor, quien tiene que validar que su trabajo o guía en clases es fructífero en su ausencia.

Estos antecedentes del segundo parcial preceden a los dos momentos que las autoras luego aprovecharon para evaluar a este conjunto de alumnos y tener resultados y conclusiones al término de la materia. Es vital puntualizar que casi al final del segundo semestre 2014 ya no eran 32 educandos sino 21 ; once jóvenes decidieron retirarse de su carrera, es decir, no solamente de la asignatura de inglés.

Continuando con el análisis de este grupo de discentes en su última clase de inglés en el mes de febrero del 2015, las autoras evaluaron nuevamente la pronunciación de este grupo de jóvenes, esta ocasión la docente la consideró el primer momento, fue un ensayo o preparación, estuvieron presentes 21 alumnos, los estudiantes no sabían que ese día iban a tener esta actividad diferente en el aula de clases, la idea fue precisamente no advertirlos para ver en realidad cómo había mejorado su pronunciación o tal vez reconocer que no hubo ningún avance fonético o que la capacidad de expresarse en forma oral es baja.

El ejercicio fue formar 10 pares de alumnos, uno trabajó con la docente recordando que asistieron aquel día 21 discentes. Tuvieron 15 minutos de práctica entre pares, el contenido fue un banco de preguntas que abarcaba todos los temas vistos 
durante el curso las mismas que fueron resueltas con anterioridad y en casi la mitad de las interrogantes los discentes tuvieron refuerzo y asesoría de la docente días anteriores en cómo pronunciar las palabras que ella consideraba difícil de producir, es decir fue la oportunidad de recordar, practicar o aprender.

Luego de la práctica o ensayo en dúo cada par de estudiantes interactuó frente a la docente, minuto y medio, las palabras en español no fueron aceptadas, tampoco la docente recordó a los practicantes lo que debían hacer, puesto que las instrucciones fueron claramente indicadas al inicio del ensayo. Ambos alumnos hicieron y respondieron una pregunta seleccionada al azar por la profesora quien únicamente dijo en inglés el número que le correspondía a cada uno, esta simple instrucción se convirtió en la primera acción a comprender y realizar bien por el alumno. Los resultados de esta observación están recopilados en la siguiente tabla:

Tabla 3. Descripción de los criterios de evaluación del ensayo oral y número de estudiantes asistentes objeto de estudio.

Número de alumnos Número de respuestas correctas

\begin{tabular}{|c|c|}
\hline 5 & 2 \\
\hline 3 & 6 \\
\hline 2 & 3 \\
\hline 2 & 4 \\
\hline 1 & 1 \\
\hline 1 & 8 \\
\hline 2 & 0 \\
\hline 16 & 24 \\
\hline
\end{tabular}

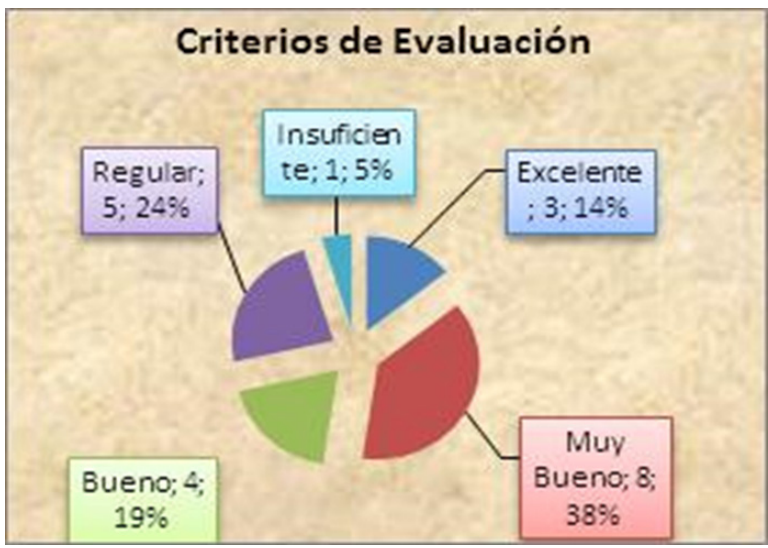

Gráfico 3.- Criterios de evaluación del ensayo oral.

Se puede observar que los resultados del primer momento de evaluación del segundo parcial, es decir del ensayo en pares en el aula, son positivos porque de los 21 alumnos, 15 están entre excelente, muy bueno y bueno (este último criterio se lo puede considerar aceptable) y esto está representado por el $71,44 \%$, en contraste con el resultado negativo que equivale al $28,57 \%$ de 6 alumnos cuyos criterios están entre regular e insuficiente.

En conclusión, quince alumnos muy buenos de veintiuno es una cantidad motivadora para la docente. Vale la pena puntualizar el comportamiento o acciones de los alumnos en este proceso de ensayo, durante su práctica en pares las autoras observaron que los estudiantes se desenvolvieron mejor entre sus iguales, pero lucieron distraídos, nerviosos e inseguros cuando tuvieron que participar en presencia de la facilitadora.

En el mes de febrero del 2015, respetando el cronograma académico de la Universidad Estatal de Milagro (UNEMI), tuvieron lugar los exámenes del segundo parcial o también llamados finales; aquí las autoras evaluaron por última vez a este grupo de estudiantes en forma individual y esta oportunidad la consideraron el segundo momento. El examen final de inglés es sobre 30 puntos y se lo divide en dos partes: 10 corresponden al examen oral y 20 al escrito, por tanto el análisis que se presenta a continuación es sobre los 10 puntos arriba enunciados, divididos en cinco preguntas cada una con una valoración de 2 . Los resultados de esta observación están recopilados en la siguiente tabla:

Tabla 4. Descripción de los criterios de evaluación del examen oral y número de alumnos objeto de estudio.

\begin{tabular}{|c|c|}
\hline Criterios & Número de alumnos \\
\hline Excelente (9-10) & 6 \\
\hline Muy Bueno (7-8) & 9 \\
\hline Bueno (5-6) & 2 \\
\hline Regular (3-4) & 4 \\
\hline \multirow[t]{2}{*}{ Insuficiente (0-1-2) } & 0 \\
\hline & 21 \\
\hline
\end{tabular}

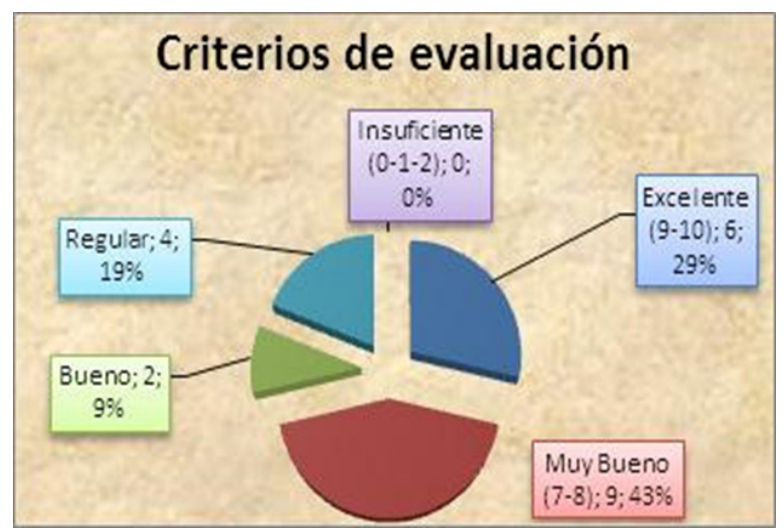


Se puede observar que los resultados de ese segundo momento final de evaluación son altamente positivos porque de los 21 alumnos de Primero de Ingeniería en Sistemas, 17 están entre excelente, muy bueno y bueno (este último criterio se lo puede considerar aceptable) y esto está representado por el $80,95 \%$, en contraste con el resultado negativo que equivale al $19,05 \%$ de 4 alumnos cuyo criterio es regular y el puntaje insuficiente es totalmente ausente.

\section{Conclusiones}

Se determina que, diecisiete alumnos muy buenos de veintiuno, es una cantidad favorable para la docente, porque significa que su estrategia funcionó, los resultados la validan. El trabajo autónomo con libertad, responsabilidad y dentro del nivel académico del discente, enriquece su experiencia de aprender una lengua extranjera, en este caso la inglesa, porque comprueba que es posible mejorar, transformarse, adquirir las destrezas de un idioma extranjero poco a poco y comprender que los errores son parte de su proceso de aprendizaje sin que ellos influyan negativamente, es decir que el alumno piense que "no puede" o que solo posee cualidades para receptar y producir en su lengua materna.

Existen varias metodologías o formas de cómo enseñar y aprender inglés desde la tradicional o clásica, que implica un conjunto de alumnos dentro del aula de clases guiados o dirigidos por su profesor donde ambos tienen objetivos que cumplir, profundamente relacionados, las clases particulares o personales que se enfocan en las necesidades o expectativas del cliente con el propósito de aprender, repasar o practicar la lengua inglesa de una forma más exclusiva, hasta los actuales cursos en línea que prometen flexibilidad en cuanto al tiempo, tutores virtuales sincrónicos y asincrónicos y dominio del idioma inglés en poco tiempo.

Dichas posibilidades son válidas, ya que las personas enseñan y aprenden de distintas maneras, lo importante es tomar conciencia de cuán valioso es desenvolverse en otro idioma porque puede ser limitante en el plano laboral, académico, incluso social y personal, solamente receptar y producir en la lengua materna. Si asociamos a profesor y estudiante, se dice que el segundo es reflejo del primero; podría ser, tal vez no. En cuanto a esta investigación se presentan los siguientes resultados:

a) La presentación y práctica de los fonemas acompañados por el docente, son solo un paso o procedimiento en el lapso dispuesto al aprendiza- je del Inglés, en este caso pronunciación elemental A1, porque en esta investigación lo muestra el resultado de los 15 alumnos, quienes fracasan en el segundo tema del reactivo tomado en el primer parcial. Sin embargo, 15 alumnos exitosamente responden al primer tema del mencionado examen, por tanto las autoras prefieren definirlo como el starting point de la estrategia aplicada en esta investigación que automáticamente, lleva a evaluar y aceptar las debilidades del grupo y tomar acciones correctivas al respecto.

b) El trabajo autónomo guiado, esto es con tareas específicas de acuerdo al nivel de Inglés que se está estudiando, funciona, así el alumno se sienta presionado por el profesor, lo corrobora el 71,44 $\%$ de los resultados del ensayo oral y sobretodo el $80,95 \%$ de los resultados del examen oral representando a 17 alumnos cuya performance fue desde lo excelente hasta lo aceptable y no hubo ningún estudiante insuficiente.

Desde los primeros encuentros con los integrantes de este grupo de Primero de Sistemas, las autoras notaron un gran potencial que tenía que aprovecharse; se hizo el mejor y mayor de los esfuerzos, la prueba de esto es que una alumna aprobó con excelente, dieciocho con muy bueno, y dos estudiantes reprobaron la materia. Si bien es cierto las notas finales es el conjunto de todas las destrezas desarrolladas y ejercicios aplicados en el lapso que duró el semestre, sin embargo la docente está completamente convencida que la habilidad fonética que tiene este grupo de alumnos, hablando en términos generales, colaboró muchísimo como fortaleza para ellos y facilitó el trabajo de la docente.

Se reconoce que se pudo haber sacado más provecho de estos jóvenes, cuyas mentes y aspiraciones por aprender están frescas y latentes, con más razón si recién se insertaron a la vida universitaria que es la antesala de preparación para la laboral; lo importante es que estos educandos y la docente ya tienen la experiencia que trabajar juntos y a veces por separado, la paciencia, autoestima, confianza en sí mismos, comprensión de cumplir objetivos a corto, mediano y largo plazo permitirán desenvolverse en una y más lenguas extranjeras.

\section{Referencias Bibliográficas}

1. Cieri, L y Barbeito M. [Online].; 2000 [cited 2015 6 20. Available from: https://www.unrc.edu.ar/publicar/cde/05/Cieri.htm.

\section{Latham-Koening C OCySP. American English} File1. Second Edition ed. Press OU, editor. Lon- 
don; 2013.

3. Oslina R. Factores que intervienen en el aprendizaje de una segunda lengua. Revista digital I E Investigación y Educación. 2011;(9).

4. Córdoba Cubillo Patrica - Coto Keith, Rossina - Ramírez Salas, Marlene. La comprensión auditiva: definición, importania, características, procesos, materiales y actividades. Revista Electrónica "Actualidades Investigativas en Educación". 2005.

5. Navas Montes Yoaniker, Real Poveda Iliana, Pacheco Mendoza Silvia, Mayorga Albán Amalin. Los Procesos de Enseñanza y Aprendizaje del Idioma Inglés a través de los Entornos Virtuales de Aprendizaje. Ciencia UNEMI. 2015 Apr; 8(13).

6. Latham-Koening C, Oxenden C, y Seligson P. American English File 1A Multi-Pack Student's Book - Workbook Cambridge , editor. London: Oxford University Press; 2013.

7. M NL. Actividades para desarrollar la comprensión auditiva y audiovisual en el aula. Revista de enseñanza ELE. 2010.

8. Fuentes Pedro \& Samaniego Eva. Los sistemas de enseñanza de inglés para fines específicos basados en el aprendiente. Revista Científica UNED. 2003; 6(2).

9. Valenzuela Manzanares J. Lingüística contrastiva inglés-español: una vision general. Revista Electrónica Interuniversitaria de Formación del Profesorado. 2010.

10. Lilian Gómez \& María Sandoval. Comprensión auditiva en inglés como L2. Revista de Linguistica Teórica y Aplicada. 2012;(8).

11. Romanelli S. Integración de la Pronunciación con las habilidades de expresión oral y expresión auditiva en el aula. Nexos. 2009.

12. Press CU. Cambridge English Language Assessment. [Online]. [cited 201501 20. Available from: http://www.cambridgeenglish.org/es/exams/ cefr/.

13. Real Poveda, lliana \& Navas Montes, Yonaiker. Estrategia para el aprovechamiento de un entorno virtual en el aprendizaje de una segunda lengua. Revista Ciencias Pedagógicas e Innovación. 2015.

14. Richard Clement, Douglas Fleming, Jennifer John. L2 Students' Willingness to Communicate Pronunication in the ESL Classroom Adult lear- ners. 2012. http://www.teslontario.net/uploads/ publications/researchsymposium/ResearchSymposium2012.pdf.

15. Guba, E.G. \& Lincoln, Y.S. http://www.gdufs. biz/10-guba_lincoln_94.pdf. [Online]. Available from: http://www.gdufs.biz/10-guba_lincoln_94. pdf.

16. Stake RE. Strategies of Qualitative Inquiry. London, New Delhi ed. London, New Delhi: London, New Delhi; 1994.

17. Derwin, Tracedy M - Lori G., Diepenbroek \& Jennifer, Foote. How well do General-Skills ESL Texbooks Address Pronunciation? TESL Canada Journal. 2012 Apr: p. 2.

18. learning. TiocsgfpP. http://repositorio.utp.edu. co/dspace/bitstream/11059/4549/1/42154P654. pdf. [Online]. 2015; 2014 [cited 201509 15. Available from: http://repositorio.utp.edu.co/dspace/ bitstream/11059/4549/1/42154P654.pdf.

19. Maridueña Macancela, Jacqueline \& Ledesma Acosta, Brigita. Orientación del Trabajo Independiente y el Uso de las TIC. Revista Científica Ciencia - UNEMI. 2015.

20. Maridueña Macancela J. Recursos Interactivo Tecnológicos y su repercusión en el Interaprendizaje del Idioma Inglés. RES NON VERBA. 2014. 